\title{
A Coupled Model for Wave Run-up Simulation
}

\author{
Iryanto $^{1, *}$ and S.R. Pudjaprasetya ${ }^{2}$ \\ ${ }^{1}$ Informatics Department, Politeknik Negeri Indramayu, Indramayu, Indonesia. \\ ${ }^{2}$ Mathematics Department, Faculty of Mathematics and Natural Sciences, Institut \\ Teknologi Bandung, Bandung, Indonesia.
}

\begin{abstract}
Simplified models like the shallow water equations (SWE) are commonly adopted for describing a wide range of free surface flow problems, like flows in river$\mathrm{s}$, lakes, estuaries, or coastal areas. In the literature, numerical methods for the SWE are mostly mesh-based. However, this macroscopic approach is unable to accurately represent the complexity of flows near coastlines, where waves nearly break. This fact prompted the idea of coupling the mesh-based SWE model with a meshless particle method for solving the Euler equations. In a previous paper, a method to couple the staggered scheme SWE and the smoothed particle hydrodynamics (SPH) Euler equations was developed and discussed. In this article, this coupled model is used for simulating solitary wave run-up on a sloping beach. The results show strong agreement with the experimental data of Synolakis. Simulations of wave overtopping over a seawall were also performed.
\end{abstract}

AMS subject classifications: 76M28, 76M10, 76B25

Key words: smoothed particle hydrodynamics, staggered conservative scheme, solitary wave runup, wave overtopping.

\section{Introduction}

Determination of wave run-up characteristics and possible flooding scenarios are important factors in the design of coastal protection structures. In recent years, wave run-up has been investigated experimentally, theoretically and numerically. For experimental studies, one can consult $[16,17]$, for instance.

The shallow water equations are a widely used model for describing wave run-up characteristics. Grid-based numerical schemes solving the shallow water equations are abundant in the literature. However, wave run-up is a physically complex phenomenon, especially when it involves wave breaking. In this paper, we combine a numerically efficient grid-based scheme with a meshless SPH method. The coupling procedure between staggered grid SWE scheme and the SPH computations developed in [4] is used here. The

\footnotetext{
*Corresponding author. Email addresses: iryanto.math@yahoo.com (Iryanto), sr_pudjap@math.itb.ac.id (S. R. Pudjaprasetya) 
staggered scheme is implemented on a large flat domain, whereas SPH computations focus on the shoreline or close to off-shore structures, where a complex description of the free-surface is required.

In research related to wave run-up, the experimental data measuring solitary wave run-up on a sloping beach as recorded by Synolakis in $[16,17]$ serves as the canonical benchmark test. Here, we present simulations of solitary waves climbing up a sloping beach calculated using three different numerical scenarios: the staggered SWE scheme, the SPH method, and the coupled model. For each case, the run-up height is recorded and compared with the aforementioned experimental data.

In Section 2, governing equations for free surface flows (the shallow water equations and the Euler equations), along with their corresponding numerical methods (a staggered grid approach and an SPH approach, respectively), are reviewed. Moreover, a coupled model of SWE and Euler models is discussed. In Section 3, simulation results for solitary wave run-up on a sloping beach are computed using each of these models. Section 4 focuses on an application of the coupled model to simulate wave overtopping of a seawall.

\section{Numerical models}

In this section, we discuss three models: the staggered grid scheme for the shallow water equations model, the SPH method for the Euler model, and a coupled model combining those two methods.

\subsection{Shallow water model}

Consider the following shallow water model, which holds for relatively long waves in a shallow region:

$$
\begin{aligned}
& h_{t}+(h u)_{x}=0, \\
& u_{t}+u u_{x}+g h_{x}=0 .
\end{aligned}
$$

In the above equations, $u(x, t)$ denotes the depth-averaged horizontal velocity, $h(x, t)$ denotes the total water layer thickness such that $h(x, t)=\eta(x, t)+d(x)$, where $\eta(x, t)$ denotes the free surface elevation and $d(x)$ describes the depth of the bottom topography relative to the undisturbed free surface height (Figure 1). Here, we consider a staggered

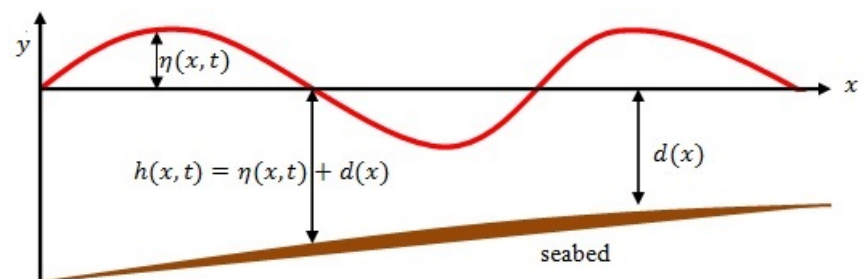

Figure 1: Sketch of fluid domain for SWE model. 
conservative scheme for solving the above shallow water equations, for which a discrete formulation of (2.1)-(2.2) is given by

$$
\begin{gathered}
\frac{h_{i}^{n+1}-h_{i}^{n}}{\Delta t}=-\left(\frac{{ }^{*} h_{i+\frac{1}{2}}^{n} u_{i+\frac{1}{2}}^{n}-{ }^{*} h_{i-\frac{1}{2}}^{n} u_{i-\frac{1}{2}}^{n}}{\Delta x}\right), \\
\frac{u_{i+\frac{1}{2}}^{n+1}-u_{i+\frac{1}{2}}^{n}}{\Delta t}=-\frac{1}{\bar{h}_{i+\frac{1}{2}}}\left(\frac{\bar{q}_{i+1}{ }^{*} u_{i+1}-\bar{q}_{i}^{*} u_{i}}{\Delta x}-u_{i+\frac{1}{2}} \frac{\bar{q}_{i+1}-\bar{q}_{i}}{\Delta x}\right)-g\left(\frac{\eta_{i+1}^{n+1}-\eta_{i}^{n+1}}{\Delta x}\right),
\end{gathered}
$$

where

$$
\bar{h}_{i+\frac{1}{2}}=\frac{h_{i}+h_{i+1}}{2}, \quad \bar{q}_{i}=\frac{q_{i+\frac{1}{2}}+q_{i-\frac{1}{2}}}{2}, \quad q_{i+\frac{1}{2}}={ }^{*} h_{i+\frac{1}{2}} u_{i+\frac{1}{2}} .
$$

Upwind approximations for ${ }^{*} h$ and ${ }^{*} u$ are given by

$$
* h_{i+\frac{1}{2}}=\left\{\begin{array}{cl}
h_{i}, & \text { if } u_{i+\frac{1}{2}}^{n} \geq 0, \\
h_{i+1}, & \text { if } u_{i+\frac{1}{2}}^{n}<0,
\end{array} \quad * u_{i}= \begin{cases}u_{i-\frac{1}{2}}, & \text { if } \bar{q}_{i} \geq 0, \\
u_{i+\frac{1}{2}}, & \text { if } \bar{q}_{i}<0 .\end{cases}\right.
$$

A more detailed discussion of this staggered scheme can be seen in [13-15].

\subsection{Euler model}

When applying a smoothed particle hydrodynamics (SPH) approach to the numerical solution of the Euler equations, the fluid domain is considered as a collection of fluid particles. The dynamics of these particles are governed by the Euler equations

$$
\begin{aligned}
& \frac{D \rho}{D t}=-\rho \nabla \cdot \mathbf{u}, \\
& \frac{D \mathbf{u}}{D t}=-\frac{1}{\rho} \nabla p+\mathbf{g}, \\
& \frac{D \mathbf{x}}{D t}=\mathbf{u},
\end{aligned}
$$

where $\mathbf{x}(t)$ denotes the position and $\mathbf{u}(t)$ denotes the velocity of each particle. The parameter $\rho$ is the fluid density, $p$ is pressure, $\mathbf{g}$ is the acceleration due to gravity, and $D / D t$ indicates the material derivative.

We apply the SPH method to solve (2.6)-(2.8). In this method, we calculate the position $\mathbf{x}_{i}$ and velocity $\mathbf{u}_{i}$ of each of the fluid's particles at every time step, where the index $i$ distinguishes the individual particles. Here, the standard SPH formulation as described in [10] and [7] will be used.

In the method, a weighting function or kernel $W\left(\mathbf{x}_{i}-\mathbf{x}_{j}, \kappa\right)$ is used to interpolate a function $\psi(\mathbf{x})$ and its spatial derivative. In discrete form, this interpolation at a point $\mathbf{x}_{i}$ can be written as 


$$
\psi\left(\mathbf{x}_{i}\right)=\sum_{j} \psi_{j} W\left(\mathbf{x}_{i}-\mathbf{x}_{j}, \kappa\right) V_{j}
$$

where $\kappa$ and $V_{j}$ represent the smoothing length of the kernel and the volume of particle $j$, respectively. Here $V_{j}=m_{j} / \rho_{j}$, where $m_{j}$ and $\rho_{j}$ are the mass and density of the particle $j$. The summation is executed for all particles $j$ within the compact support region of the kernel.

In related research, the most commonly used cubic spline kernel is

$$
W(r, \kappa)=\beta \begin{cases}1-\frac{3}{2} R^{2}+\frac{3}{4} R^{3}, & 0 \leq R \leq 1 \\ \frac{1}{4}(2-R)^{3}, & 1 \leq R \leq 2 \\ 0, & R \geq 2\end{cases}
$$

where $\beta$ is the normalization parameter for two-dimensional space defined by $10 /\left(7 \pi \kappa^{2}\right)$, $R=r / \kappa$ denotes the non-dimensional distance between particles $i$ and $j$, and $r$ is the distance between particles. The particle approximation for the spatial derivative of a function $\psi(\mathbf{x})$ for particle $i$ is given as

$$
\nabla \cdot \psi\left(\mathbf{x}_{i}\right)=-\sum_{j} \psi_{j} \cdot \nabla W\left(\mathbf{x}_{i}-\mathbf{x}_{j}, h\right) V_{j}
$$

where

$$
\nabla W\left(\mathbf{x}_{i}-\mathbf{x}_{j}, \kappa\right)=\nabla_{i} W_{i j}=\frac{\mathbf{x}_{i}-\mathbf{x}_{j}}{r_{i j}} \frac{\partial W_{i j}}{\partial r_{i j}}, \quad r_{i j}=\left\|\mathbf{x}_{i}-\mathbf{x}_{j}\right\| .
$$

Further, notation $\partial W_{i j} / \partial r_{i j}$ in (2.11) denotes the derivative of the kernel function.

By applying this method, we find that the SPH formulation of the Euler equations (2.6)-(2.7) is expressed as

$$
\begin{aligned}
& \frac{D \rho_{i}}{D t}=\sum_{j=1}^{N} m_{j}\left(\mathbf{u}_{i}-\mathbf{u}_{j}\right) \cdot \nabla_{i} W_{i j}, \\
& \frac{D \mathbf{u}_{i}}{D t}=-\sum_{j=1}^{N} m_{j}\left(\frac{p_{i}+p_{j}}{\rho_{i} \rho_{j}}+\Pi_{i j}\right) \nabla_{i} W_{i j}+\mathbf{g},
\end{aligned}
$$

where $p_{i}$ and $p_{j}$ are the pressure of particle $i$ and $j$, respectively. The term $\Pi_{i j}$ represents an artificial viscosity added to help achieve numerical stability, and is defined using

$$
\Pi_{i j}= \begin{cases}\frac{-\alpha \bar{c}_{i j} \mu_{i j}}{\bar{\rho}_{i j}}, & \mathbf{u}_{i j} \cdot \mathbf{x}_{i j}<0 \\ 0, & \text { otherwise }\end{cases}
$$

with

$$
\mu_{i j}=\frac{\kappa_{i j} \mathbf{u}_{i j} \cdot \mathbf{x}_{i j}}{r_{i j}^{2}+\left(0.1 \kappa_{i j}\right)^{2}}, \quad \mathbf{u}_{i j}=\mathbf{u}_{i}-\mathbf{u}_{j}, \quad \mathbf{x}_{i j}=\mathbf{x}_{i}-\mathbf{x}_{j},
$$




$$
\bar{c}_{i j}=0.5\left(c_{i}+c_{j}\right), \quad \bar{\rho}_{i j}=0.5\left(\rho_{i}+\rho_{j}\right), \quad \kappa_{i j}=0.5\left(\kappa_{i}+\kappa_{j}\right) .
$$

The coefficient $\alpha$ is a constant, and $c_{i}$ and $c_{j}$ represent the speed of sound of particles $i$ and $j$ respectively. In the work of Padova, et al., [12], the effect of this artificial viscosity is analyzed through a series of six different experiments, and the results of SPH computations are compared to experimental data. In the current article, the constant $\alpha$ is chosen as $\alpha=0.025$.

We note that in the SPH method, the fluid is treated as weakly compressible by employing an equation of state. Here, the following equation of state proposed by Monaghan [9] is used:

$$
p(\rho)=\frac{c_{0}^{2} \rho_{0}}{\gamma}\left[\left(\frac{\rho}{\rho_{0}}\right)^{\gamma}-1\right],
$$

where $\gamma=7, \rho_{0}=1000 \mathrm{~kg} \mathrm{~m}^{-3}$ is the reference density, and $c_{0}=c\left(\rho_{0}\right)=\left.\sqrt{(\partial p / \partial \rho)}\right|_{\rho_{0}}$ represents the speed of sound at the reference density. As for the time integration used to solve (2.8), the leapfrog scheme is used.

\subsection{Coupled model}

In this section, we review the main ideas behind a coupled model formulated with the purpose of combining the efficiency of the SWE staggered scheme with the physical accuracy of SPH computations. A more detailed description of this method can be found in [4]. Our scenario involves implementing the SWE staggered scheme on the large domain while applying the Euler SPH method near the shoreline area. A buffer zone is defined between these two areas, connecting the shallow water area on the upstream side with the SPH shoreline area on the downstream side. In this buffer zone, information is exchanged between the two models.

In the SWE, there are two dependent variables (horizontal velocity $u(x, t)$ and total water layer thickness $h(x, t)$ ), whereas in SPH, we deal with the positions $\mathbf{x}_{i}(t)=$ $\left(x_{i}(t), y_{i}(t)\right)$ and velocities $\mathbf{u}_{i}(t)=\left(u_{i}(t), v_{i}(t)\right)$ of particles. Clearly, coupling those two models is not a trivial task. We adopt the idea of coupling SPH with Boussinesq equations as described in $[5,11]$, and implement them to couple the SWE with SPH.

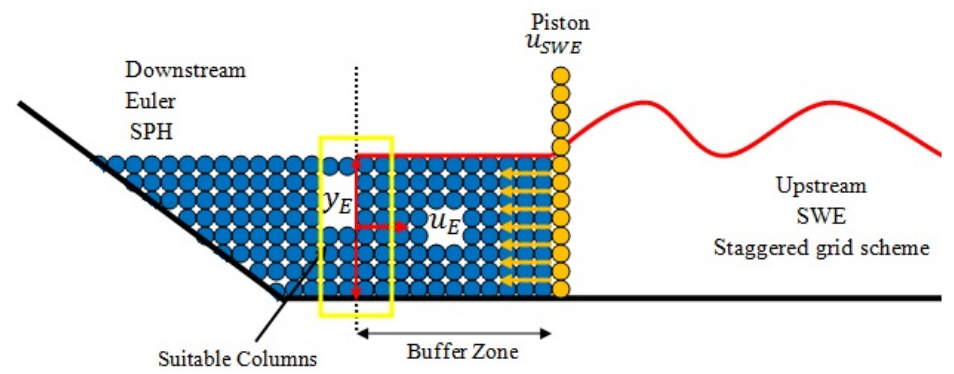

Figure 2: Sketch of SWE domain and Euler SPH domain with a buffer area in the coupled model. 
Consider Figure 2, in which the physical domain is split into two regions of implementation: an upstream part where the SWE are to be implemented, and a downstream part where the Euler equations are to be implemented. In the middle, there is a buffer zone where results of both models are computed. Particles attached to the right side of this buffer zone stay connected, playing the role of a wavemaker (piston) that transfers information from the SWE region into the SPH region. This "piston" moves with horizontal velocity $u_{S W E}(t)$. In the other direction, SPH region information is transferred back into the SWE region using particles on the left side of the buffer zone, indicated by the yellow rectangle in Figure 2. At each time $t$, the maximum value of $y_{i}(t)$ over all particles in the rectangle is computed. This maximum height, together with the averaged horizontal velocity, becomes the left boundary condition for the SWE computation in the upstream part. We note here that the buffer zone should be defined so as to be long enough to accommodate piston movement. In this way, information is communicated between the SWE and SPH regions at all times. In [4], the above procedure has been tested for simulating the propagation of monochromatic waves, but similar simulations of solitary wave propagation have not yet been investigated. In the next section, we apply this coupled model to simulate solitary wave run-up on a sloping beach.

Since the coupled model is a combination of the SWE staggered conservative scheme (upstream) and the Euler SPH method (downstream), the discrete formulations of the SPH approach (2.12)-(2.13) and the staggered scheme (2.3)-(2.4) are used. In the buffer zone, information transfer between the SWE model and the Euler model is conducted. In our previous paper [4], the scheme was successfully used for testing the propagation of monochromatic waves.

\section{Solitary wave run-up on a simple beach}

Synolakis et al. in $[16,17]$ studied solitary wave run-up on a sloping beach both experimentally and analytically. His experimental results have become the primary benchmark test for numerical methods of solitary wave run-up. In this section, we use the experimental data for solitary run-up by Synolakis (obtained from [18]) to check the validity of the numerical methods.

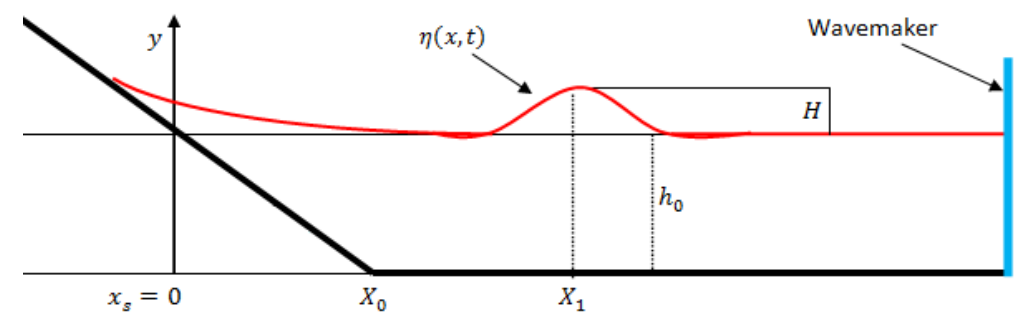

Figure 3: Sketch of solitary wave run up on a simple beach.

A sketch of the problem is depicted in Figure 3. The experiment was carried out in a 
wave tank with dimensions of $37.73 \mathrm{~m}$ in length, $0.39 \mathrm{~m}$ in width, and $0.61 \mathrm{~m}$ in height. Solitary waves were generated by a wave generator, and an inclined beach was constructed from a smooth surface. In Figure $3, \eta(x, t)$ indicates the free surface elevation, $H$ is the solitary wave amplitude, and $X_{1}=x_{0}+(1 / k) \cosh ^{-1}(\sqrt{20})$ gives the initial position of the wave crest. For the bottom topography, $h_{0}$ indicates the uniform depth of the wave tank, from which the incline begins at position $X_{0}=19.85$.

For our simulations, we apply three different models: the staggered scheme for SWE, the SPH method for solving the Euler equations, and the coupled method. For simulations of wave run-up, we used an initial solitary wave of amplitude $H$ over a water depth $h_{0}$ defined by

$$
\begin{gathered}
\eta(x, 0)=\frac{H}{h_{0}} \operatorname{sech}^{2}\left(k\left(x-X_{1}\right)\right) \quad \text { with } \quad k=\sqrt{3 H / 4 h_{0}}, \\
u(x, 0)=-\sqrt{g\left(H+h_{0}\right)} \frac{\eta(x, 0)}{h_{0}+\eta(x, 0)} .
\end{gathered}
$$

Note that the choice of $u(x, 0)$ is important in order to generate a solitary wave which propagates to the left. The run-up simulation is conducted over a sloping beach as depicted in Figure 3, with topography defined by

$$
d(x)=\left\{\begin{array}{cc}
-\frac{h_{0}}{X_{0}} x & x \leq X_{0} \\
-h_{0} & x>X_{0}
\end{array}\right.
$$

For the remainder of the article, units will be omitted when displaying results for various physical quantities, with the convention that SI units are implied.

\subsection{Shallow water model}

In this subsection, solitary wave run-up over a sloping beach is simulated using the staggered conservative SWE scheme. The initial solitary wave hump has an amplitude $H=0.3$ over a flat water depth $h_{0}=1$. Here $u(L, t)=0$ is taken as the right boundary condition. We note that this will not interfere with the run-up process since $L$ is quite far from the initial hump, which is left-running. Computations are conducted using a spatial grid spacing $\Delta x=0.028$ and time step $\Delta t=8 \times 10^{-4}$. Surface elevations at subsequent time steps are plotted in Figure 4.

During propagation, the surface wave profiles shown in Figure 4 (a) and (b) were somewhat different from the experimental data, but the run-up height as shown in Figure 4 (c) and (d) were in good agreement with experimental data. The run-up height observed in this simulation was 0.40384 .

As shown in Figure 4 due to the nonlinearity in SWE, the computed wave have steeper front compare to those of the experimental wave. However, both waves have the same run-up height. Further comments on this aspect, as recommended by extensive literature on run-up events, the run-up height as one of the main run-up characteristics, can already be obtained using linear models. This recommendation is based on thorough study that involves a series of laboratory experiment in [16], also analytic and numerical studies in $[2]$. 

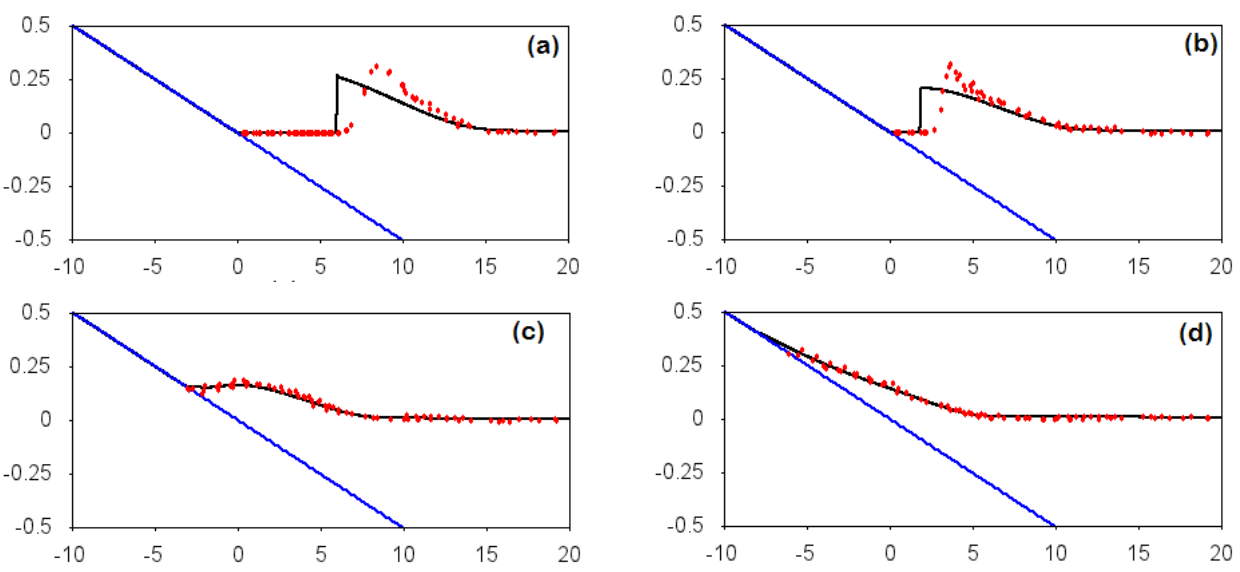

Figure 4: Solitary wave run-up calculated using SWE (solid line) together with experimental data (dots) at times (a) $t=15 / t_{0}$, (b) $t=20 / t_{0}$, (c) $t=25 / t_{0}$, and (d) $t=30 / t_{0}$, with $t_{0}=\sqrt{g / h_{0}}$.

\subsection{Euler model}

The SPH simulation was initialized by filling the domain with fluid particles spaced uniformly with distance $\kappa=0.028$. Particles located along the bottom topography were set to be fixed at all times. Particle densities were assumed to be depth-dependent according to the following relation proposed by [8]:

$$
\rho(z)=\rho_{0}\left(1+\frac{\rho_{0} g\left(h_{0}-z\right)}{\left(c_{0}^{2} \rho_{0} / \gamma\right)}\right)^{1 / \gamma}
$$

where $c_{0}$ denotes the speed of sound at the reference density.

In this SPH simulation, a solitary wave is generated by moving a piston. The piston itself is just a collection of water particles attached on the right boundary that can be moved horizontally. Letting $x_{p}(t)$ denote the piston position at time $t$, as recorded in $[6,8]$ the following piston motion will generate a left running solitary wave of amplitude $H$ over a flat depth $h_{0}$ :

$$
x_{p}(t)=x p-\frac{\left(H / h_{0}\right)}{k}\left(\frac{\tanh (k C t)}{1+\left(H / h_{0}\right)\left(1-\tanh ^{2}(k C t)\right)}\right), \quad C=\sqrt{g\left(H+h_{0}\right)} .
$$

Over a flat water depth $h_{0}=1$, the solitary wave of amplitude $H=0.3$ is generated by moving a piston using (3.5). For computations, we use $\Delta t_{\mathrm{SPH}}=2 \times 10^{-4}, c_{0}=16.5$, $\alpha=0.025$, and the commonly used SPH parameter values of $\rho_{0}=1000$ and $\gamma=7$. These parameters were also used in SPH computations as recorded in [1,3,7-9].

Numerical results from the SPH model are presented in Figure 6. Good agreement with experimental data is seen during propagation, as illustrated in Figure 6 (a) and (b) as 


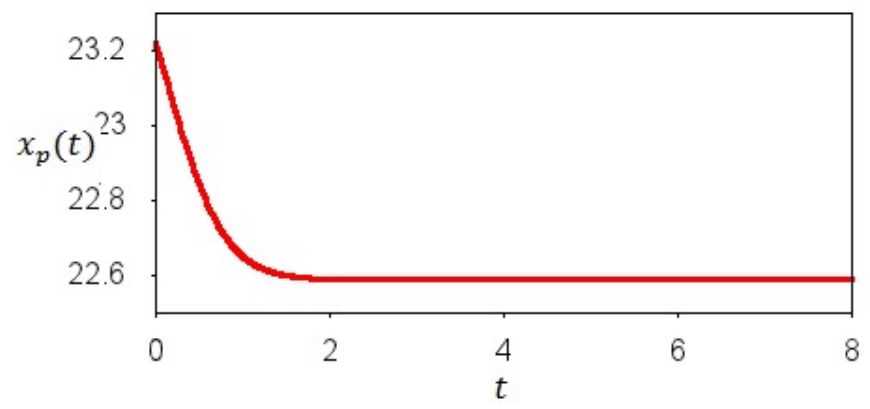

Figure 5: Piston position as a function of time for generating a solitary wave with amplitude $H$ over a flat depth $h_{0}$.
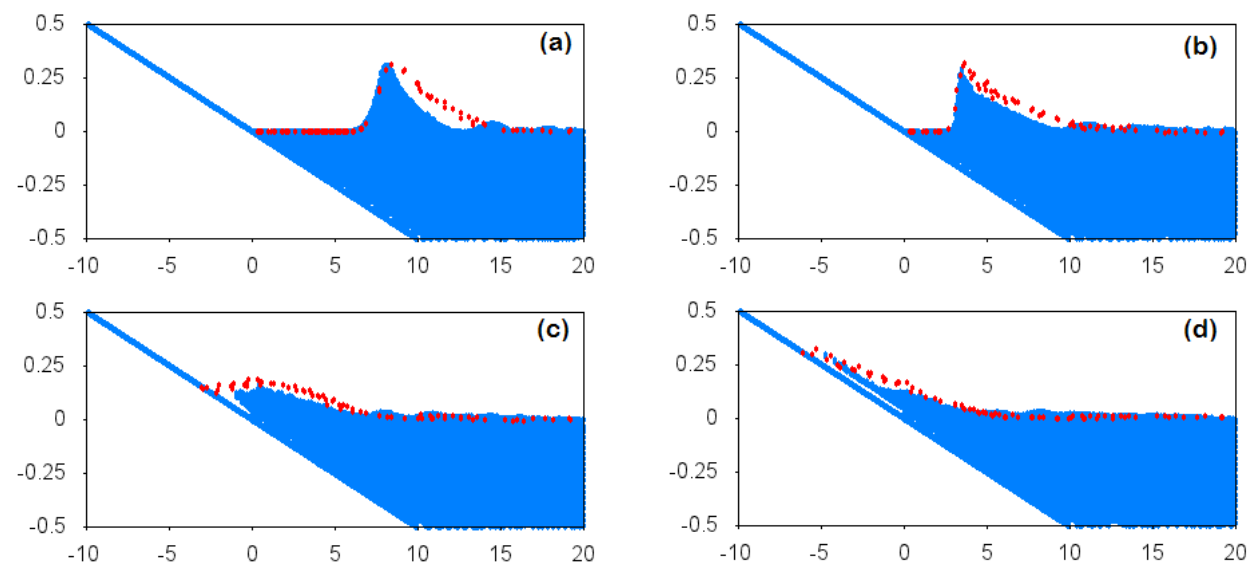

Figure 6: Solitary wave run-up calculated using SPH together with experimental data (dots) at subsequent times (a) $t=15 / t_{0}$, (b) $t=20 / t_{0}$, (c) $t=25 / t_{0}$, and (d) $t=30 / t_{0}$, with $t_{0}=\sqrt{g / h_{0}}$. 
Table 1: Comparison of the run up height

\begin{tabular}{|c|c|c|}
\hline Approach & Height & Relative error \\
\hline Experiment [18] & 0.32361 & \\
\hline Shallow water model & 0.40384 & $24.79 \%$ \\
\hline Euler model & 0.299642 & $7.41 \%$ \\
\hline Coupled model & 0.34248 & $5.83 \%$ \\
\hline
\end{tabular}

well as during run-up (c) and (d). The observed run-up height was 0.299642. Clearly, the SPH results show a better agreement compared with results of the staggered SWE scheme. However, one obvious drawback of the SPH is its high computational cost. This is due to the fact that, for every iteration, one needs to compute positions $\mathbf{x}_{\mathbf{i}}$ and velocity $\mathbf{u}_{\mathbf{i}}$ of all particles. The computational task will thus be very heavy if the physical domain is large. One way to overcome this problem is to use a coupled model.

\subsection{The coupled model}

For simulations using the coupled model, parameter values are chosen to be the same as in previous simulations using the shallow water and Euler models. In the SWE region, the same initial solitary wave (3.1)-(3.2) with $H=0.3, h_{0}=1$ is used. This solitary wave propagates to the left, enters the buffer zone, and continues into the SPH domain where it eventually reaches its run-up on a sloping beach. In this simulation, SWE model results are computed over the interval $[19.8,30]$, while the Euler equations model is implemented over $[-10,21.5]$.

In Figure 7, simulation results for solitary wave run-up calculated using the coupled model and the SWE model are plotted together along with experimental data. These results demonstrate that the coupled model runs well, with surface profiles that are in good agreement with experimental data. Moreover, the solitary wave run-up estimated by the coupled model is more accurate than the result from the shallow water staggered scheme; the run-up height recorded in the coupled model was 0.34248 .

Run-up heights obtained from the three numerical models, together with experimental data as recorded in [18], are collected in Table 1. We observe that the coupled model yields the smallest relative error from among the three approaches.

\section{Simulation of wave overtopping of a seawall}

In this section, we investigate the use of the coupled model for simulation of wave overtopping of seawalls. A sketch of the physical domain is depicted in Figure 8. Simulations were conducted for two different types of seawall as illustrated in Figure 9: vertical and inclined. In these simulations, we wish to predict the height of wave overtopping for each type of seawall. 

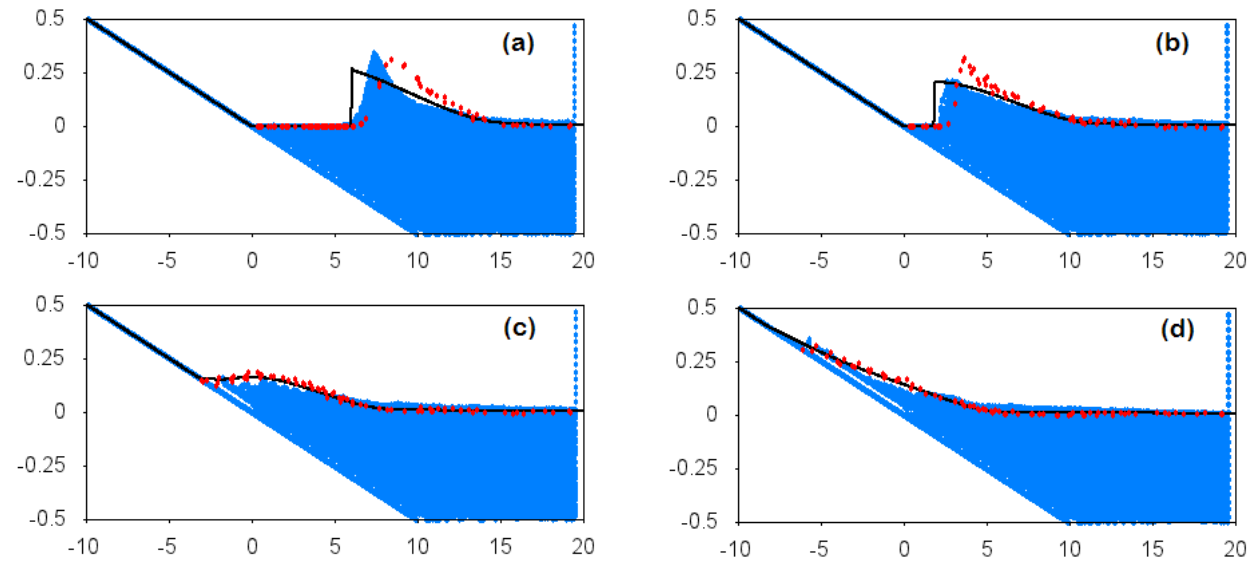

Figure 7: Numerical results for solitary wave run-up simulations using the coupled-model and SWE (solid line) with experimental data (dots) at times (a) $t=15 / t_{0}$, (b) $t=20 / t_{0}$, (c) $t=25 / t_{0}$, and (d) $t=30 / t_{0}$, with $t_{0}=\sqrt{g / h_{0}}$.

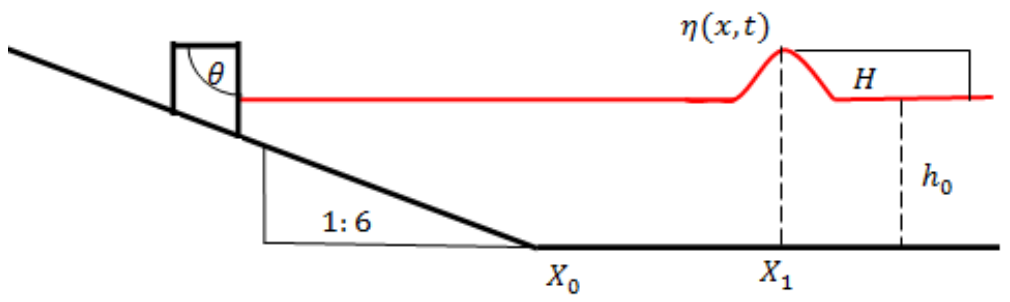

Figure 8: Sketch of fluid domain in the simulation of wave overtopping of a seawall.
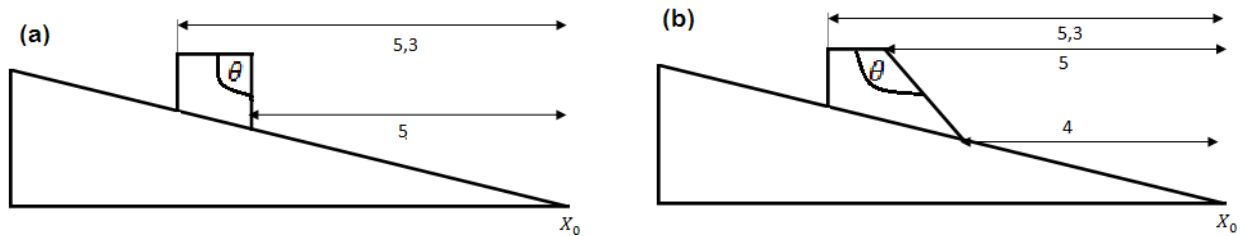

Figure 9: Dimension of the seawalls. (a) type I with $\theta=90^{\circ}$ and (b) type II with $\theta=155^{\circ}$ 

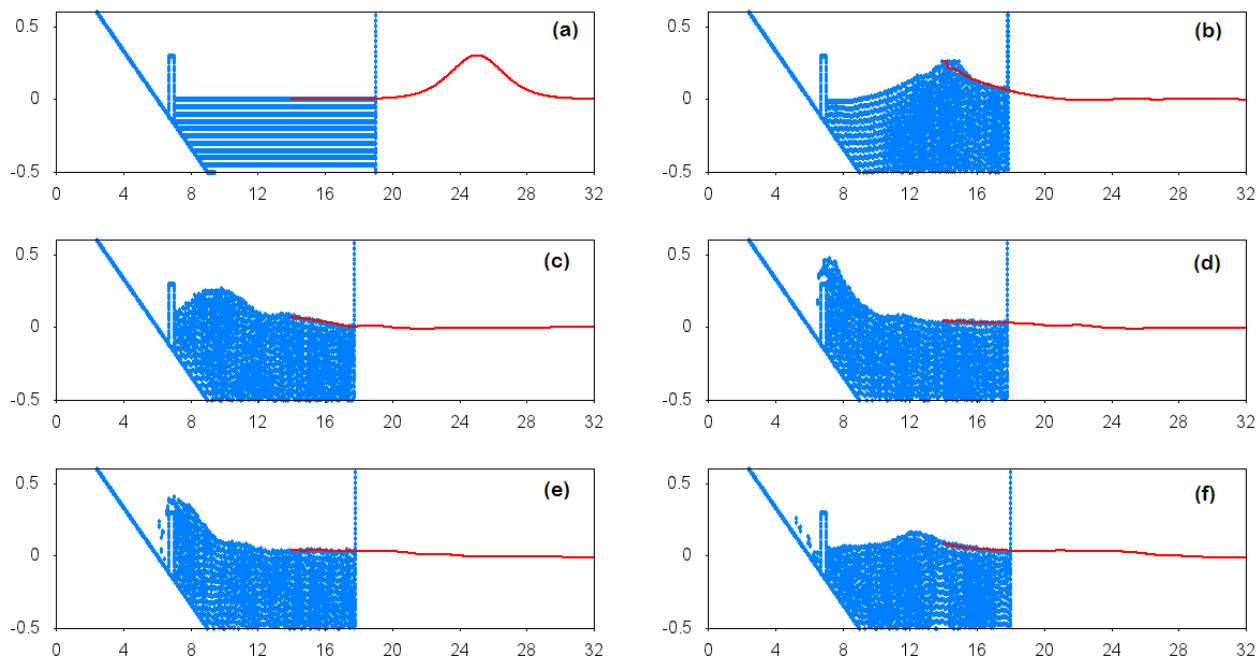

Figure 10: Wave overtopping over a Type I seawall with $\theta=90^{\circ}$ at times (a) $t=0$, (b) $t=3$, (c) $t=4.5$, (d) $t=5.5$, (e) $t=5.9$, and (f) $t=7.5$.

Here, the computational domain for the SWE model region is $[14,32]$ and the domain of the Euler region is $[0,19]$. The solitary wave initial condition used is identical to those described in previous sections. For staggered scheme computations, we use a mesh spacing of $\Delta x=0.05$, which is also used as the space between particles in the SPH computation. We set $\Delta t_{S W E}=4 \Delta t_{S P H}=2.4 \times 10^{-3}$. Simulation results are shown in Figure 10 for seawall type I, and Figure 11 for seawall type II.

Figure 10 and Figure 11 illustrate the wave impacting the seawall, causing some fluid particles to flow over the wall. In our simulations of solitary waves with height $H=0.3$, wave overtopping heights measured at the toe of seawall were observed to reach $1.33 \mathrm{H}=$ 0.408 for Type I seawalls, and $1.45 H=0.435$ for Type II seawalls.

\section{Conclusions}

We revisited a numerical model for wave run-up simulations that couples a staggered SWE scheme with a SPH implementation of the Euler equations, this time applying the model to simulate solitary wave run-up on a sloping beach. The coupled scheme was tested alongside both of the original methods - the staggered conservative scheme for SWE, and the SPH method for the Euler equations - implemented individually. All results were in a good agreement with the experimental data of Synolakis. The coupled model was indeed confirmed to strike a balance between the desirable features of both models: the accurate simulation of detailed flow characteristics achieved by the SPH Euler model on one hand, and the greater computational efficiency of the staggered SWE scheme on the other. As an additional experiment using this coupled model, we simulated wave overtopping of 

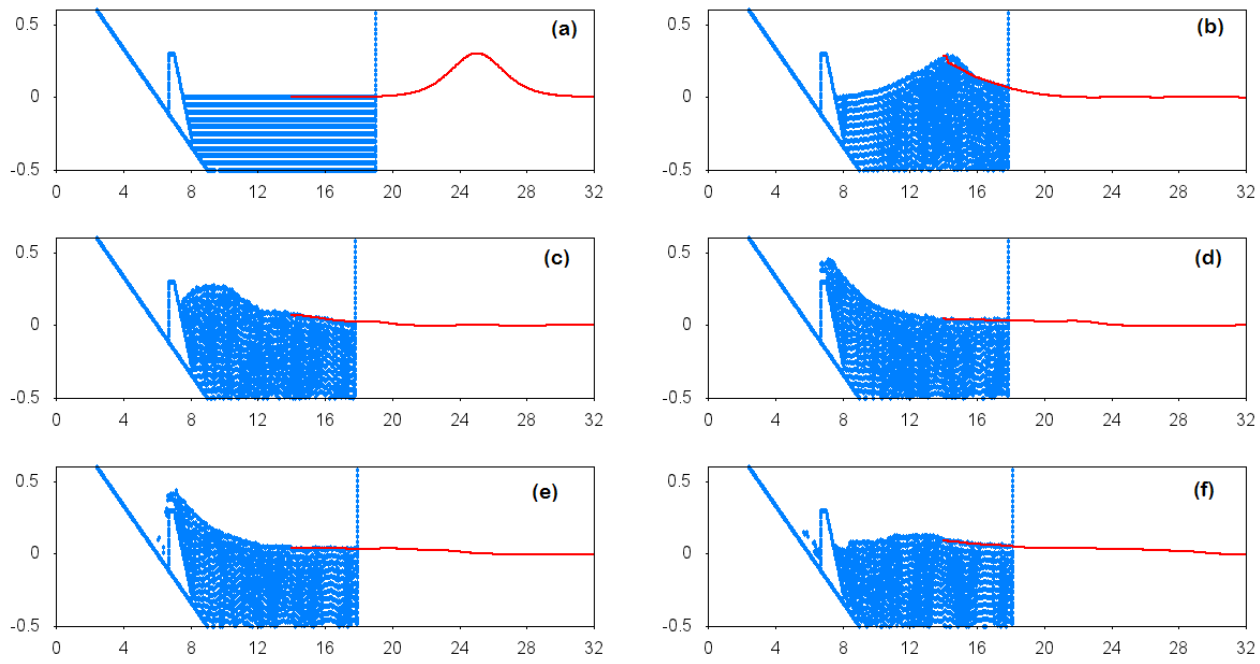

Figure 11: Wave overtopping over a Type II seawall with $\theta=155^{\circ}$ at times (a) $t=0$, (b) $t=3$, (c) $t=4.5$, (d) $t=5.5$, (e) $t=5.9$, and (f) $t=7.5$.

seawalls. These results may help to improve our understanding of the relationship between various seawall shapes and the resulting overtopping wave heights.

\section{Acknowledgment}

We wish to thank anonymous referees for useful comments that have improved the presentation of this paper. This research was supported by Institut Teknologi Bandung Desentralisasi Research Grant 2017.

\section{References}

[1] M. Becker and M. Teschner, Weakly compressible SPH for free surface flows, in Proceedings of the 2007 ACM SIGGRAPH/Eurographics symposium on Computer animation, pp. 209-217, Eurographics Association, 2007.

[2] I. Didenkulova and E. Pelinovsky, Run-up of long waves on a beach: the influence of the incident wave form, Oceanology 48, 1-6 (2008).

[3] M. Gomez-Gesteira, B. D. Rogers, A. J. Crespo, R. Dalrymple, M. Narayanaswamy, and J. M. Dominguez, SPHysics-development of a free-surface fluid solver-part 1: Theory and formulations, Computers \& Geosciences 48, 289-299 (2012).

[4] Iryanto, Shallow water - Navier-Stokes coupling method in ocean wave simulation, in International Symposium on Computational Science, pp. 56-68, 2015.

[5] C. Kassiotis, M. Ferrand, D. Violeau, B. Rogers, P. Stansby and M. Benoit, Coupling SPH with a 1-D Boussinesq-type wave model, in 6th International SPHERIC Workshop, pp. 241-247, 2011.

[6] G. Katell and B. Eric, Accuracy of solitary wave generation by a piston wave maker, J. Hydraul. Res. 40, 321-331 (2002). 
[7] G.-R. Liu and M. B. Liu, Smoothed particle hydrodynamics: a meshfree particle method. World Scientific, 2003.

[8] A. Mahmoudi, H. Hakimzadeh and M. J. Ketabdari, Simulation of wave propagation over coastal structures using WCSPH method, Journal of Marine Engineering 2, 1-13 (2014).

[9] J. J. Monaghan, Simulating free surface flows with SPH, J. Comput. Phys. 110, 399-406 (1994).

[10] J. J. Monaghan, Smoothed particle hydrodynamics, Annu. Rev. Astron. Astrophys. 30, 543-574 (1992).

[11] M. Narayanaswamy, A. J. C. Crespo, M. Gómez-Gesteira and R. A. Dalrymple, SPHysicsFUNWAVE hybrid model for coastal wave propagation, J. Hydraul. Res. 48, 85-93 (2010).

[12] D. De Padova, R. A. Dalrymple and M. Mossa, Analysis of the artificial viscosity in the smoothed particle hydrodynamics modelling of regular waves, J. Hydraul. Res. 52, 836-848 (2014).

[13] S. R. Pudjaprasetya and I. Magdalena, Momentum conservative schemes for shallow water flows, East Asian J. Appl. Math. 4, 152-165 (2014).

[14] G. S. Stelling and S. A. Duinmeijer, A staggered conservative scheme for every Froude number in rapidly varied shallow water flows, International Journal for Numerical Methods in Fluids 43, 1329-1354 (2003).

[15] G. Stelling and M. Zijlema, An accurate and efficient finite-difference algorithm for nonhydrostatic free-surface flow with application to wave propagation, International Journal for Numerical Methods in Fluids 43, 1-23 (2003).

[16] C. E. Synolakis, The runup of solitary waves, Journal of Fluid Mechanics 185, 523-545 (1987).

[17] C. E. Synolakis, E. N. Bernard, V. V. Titov, U. Kânoğlu and F. González, Standards, criteri$a$, and procedures for NOAA evaluation of tsunami numerical models: Seattle, Washington, NOAA/Pacific Marine Environmental Laboratory, tech. rep., Technical Memorandum OAR PMEL-135, 2007.

[18] The NOAA Center for Tsunami Research, Solitary wave on a canonical beach, http://nctr.pmel.noaa.gov/benchmark/Laboratory/Laboratory_ CanonicalBathymetry/index.html, accessed on 18 September 2015. 